\title{
Role of Electrolytes in the Contractile Machinery of Vascular Smooth Muscle*
}

\author{
David F. Bohr, m.D. and Patricia L. Goulet \\ Ann Arbor, Michigan
}

\begin{abstract}
$A^{1}$
LTHOUGH THE investigator who undertakes an inquiry into the cause of the elevated arterial pressure in experimental hypertension should not ignore the possibility of an elevated cardiac output, his most promising avenue of research will be in the possible causes of an increase in total peripheral resistance. In organizing his approach to this problem he must recognize that total peripheral resistance may be elevated by any one of the following, reasonably well separable mechanisms (Fig. 1): (1) an increase in neurogenic constrictor traffic to the resistance vessels; (2) an increase in circulating humoral constrictor substances; (3) an increase in vascular smooth muscle responsiveness to the normal determinants of vascular smooth muscle tone; and (4) a change in structure of the vessel wall, increasing peripheral resistance.

Of these four possible causes of increased total peripheral resistance, the one that is probably most difficult to evaluate is the increase in vascular smooth muscle responsiveness. Yet a primary basis for the current seminar on the role of salt in cardiovascular hypertension is the possibility that an altered functional electrolyte composition in vascular smooth muscle is responsible for an exaggeration in the response of the muscle to normal vasoconstrictor stimuli.

Appraisal of the validity of this theory may be made by analysis of the relative electrolyte composition of blood vessels from normotensive and hypertensive animals or by evaluation of the effectiveness of imposed alterations in electrolyte composition on the contractile process in vascular smooth muscle. The current study, which is part of a broad program aimed at describing the contractile machinery of vascular smooth muscle, falls into the latter category. The
\end{abstract}

theme of this presentation will emphasize the complexity of this problem and the superficial level of our current insight into it. The contractile process is quite like a jigsaw puzzle (Fig. 2) in which most of the pieces are not yet satisfactorily in place. These pieces consist of at least seven physiologically active ions: $\mathrm{Na}^{+}$, $\mathrm{K}^{+}, \mathrm{H}^{+}, \mathrm{Ca}^{++}, \mathrm{Mg}^{++}, \mathrm{Cl}^{-}$and $\mathrm{HCO}_{3}{ }^{-}$. A clue in properly positioning these pieces to make the final picture is the realization that there are at least four somewhat separable processes which play a role in the contraction and relaxation of the vascular smooth muscle cell. (1) Events in the cell membrane as they alter either electrochemical gradients or permeability to electrolytes and other vasoactive agents constitute the usual starting point for alterations in the contractile state of the cell. (2) A coupling process by which the influence of these membrane phenomena is transmitted to the contractile protein forms the second link in the chain of events. (3) The third process and the one responsible for the actual mechanical change is the chemomechanical transducing which is a property of the contractile protein. This change in mechanical state is presumably dependent on an energy source in organic phosphates. (4) Metabolic processes in the cell are responsible for the elaboration of these high energy phosphates.

The complexity of this picture and our current state of ignorance are emphasized by the realization that there is little if any adequate experimental evidence describing the role of any one of the physiologically active clcctrolytes in any one of these four processes. Somewhat better associations have been made between the various electrolytes and specific processes in skeletal, cardiac and even other smooth muscle

\footnotetext{
* From the Department of Physiology, University of Michigan, Ann Arbor, Michigan. This research was supported by a grant from The American Heart Association and by grant H-3576 C2 from The National Heart Institute, National Institutes of Health.
} 
EXPERIMENTAL HYPERTENSION

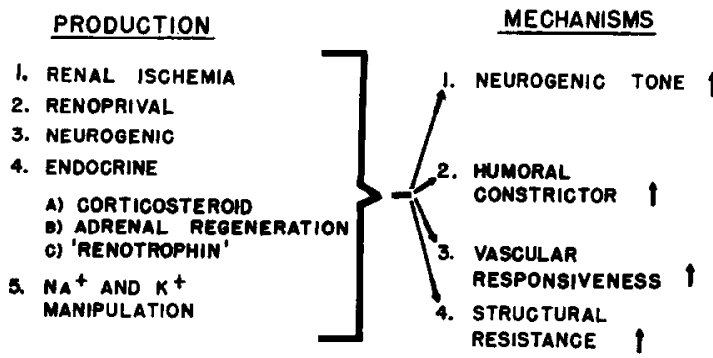

Fig. 1. Experimental hypertension; methods of production and possible mechanisms involved.

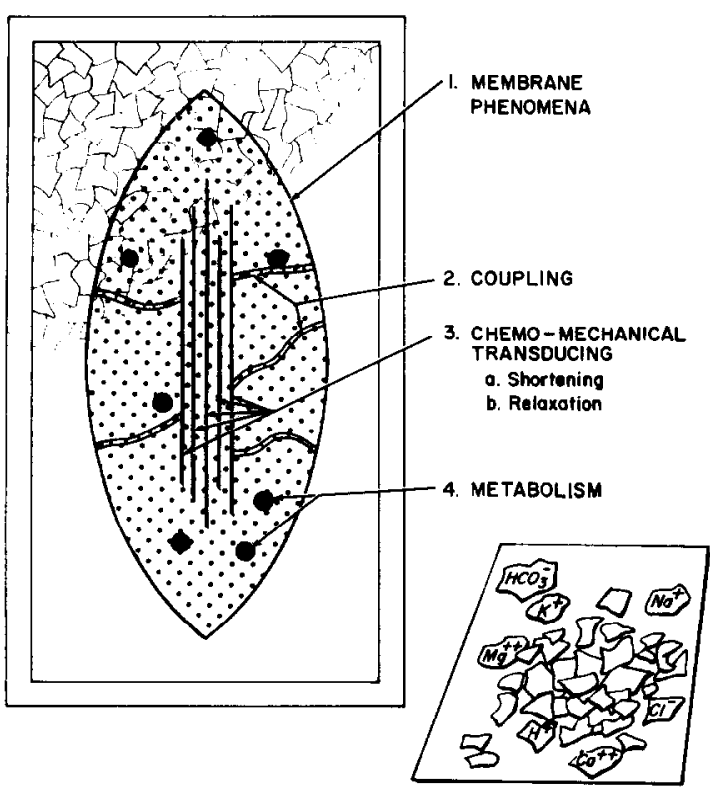

FIG. 2. Jigsaw puzzle representation of components of the contractile mechanism in a vascular smooth muscle cell. The object of the game is to fit the ion pieces (represented at the lower right of the figure) into the several processes (named at the right) which make up the contractile mechanism.

Our current impression of the role of these electrolytes in the mechanical responsc of vascular smooth muscle therefore arises largely from analogy from these other studies.

The pattern of our current approach to the problem has been to evaluate the effects of variations in the concentration of a single electrolyte on the mechanical performance of isolated vascular smooth muscle. Three objections to this type of study have been met, with varying levels of satisfaction: (1) It has been pointed out that such studies require the use of large blood vessels, the smooth muscle of which is not concerned in vascular resistance.

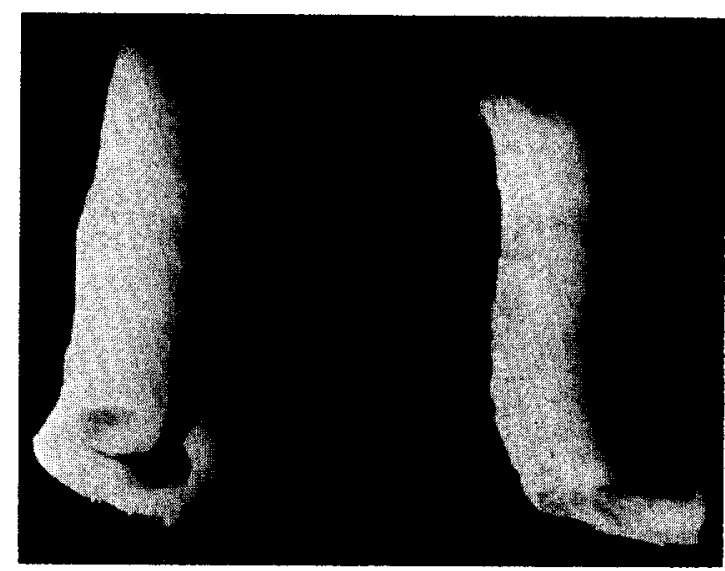

FIG. 3. Portion of aorta from normal (right) and hypertensive (left) rat showing helical strip cut from each.

We have met this objection by developing a technic which enables us to record tension from isolated vascular smooth muscle obtained from resistance vessels. (2) It is evident that the environment of the vascular smooth muscle cell in the isolated system must be different from that in vivo. It is also certain that both intraluminal and extraluminal factors in vivo must alter the response of the vascular smooth muscle cell. It must be realized that these same influences cannot be adequately controlled and therefore will superimpose their effects on that of the electrolyte being studied. Furthermore, although it is possible to measure changes in resistance in a vascular bed, it is impossible to extrapolate from these measurements definite information about active tension in the vascular smooth muscle cell. The isolated tissue therefore becomes a necessary starting point for securing this basic information. (3) The effect of a given shift in electrolyte composition in the environment of the isolated vascular smooth muscle gives little direct information related to the site of this influence in the contractilc proccss. In their prescnt form, thcrcfore, these studies are merely empirical observations from which certain interpretations can be drawn. The need for caution in making such interpretations is emphasized by the fact that certain electrolyte shifts alter more than one of the phenomena involved. An example of such a complicating situation will be presented in which it is apparent that a unidirectional shift of potassium enhances vascular response by its effect on one mechanism while it depresses vascular response by its effect on another mechanism. The effect that 


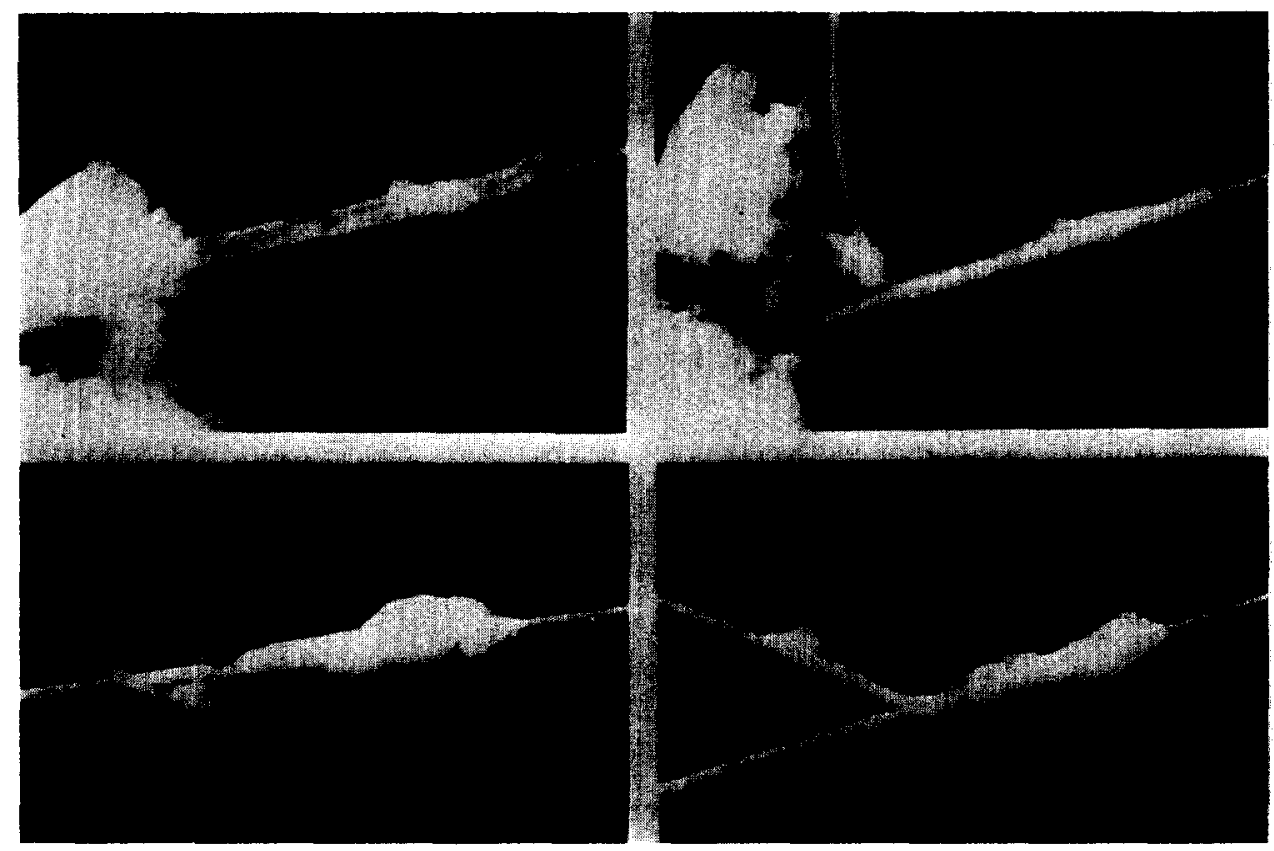

Fig. 4. Preparation of "arteriolar" strip. A, a small vessel $(250 \mu)$ is partially freed from surround. ing mesentery (rabbit). B, a thread is knotted around the proximal end of the vessel and a fine rod is threaded through the vessel from the distal end, penetrating the wall near the knot. C, rod and vessel in place in lathe. The cutting blade may be seen near the thread. The "arteriole," on the rotating rod, is guided into the cutting blade by means of this thread. D, cutting of helical strip from "arteriole" is in progress. From: BoHR, D. F., Goulet, P. and TAquini, A. C., JR. Angiology. In press. ${ }^{2}$

will be evident from this shift in potassium concentration will therefore depend on which of the processes affected is the limiting factor for contraction.

\section{Methods}

To prepare smooth muscle from large arteries for study in the isolated system, helical strips (Fig. 3) were obtained. Those illustrated are of rat aorta; those used in the current study were obtained primarily from rabbit aorta and from dog carotid. The strips were mounted in muscle chambers in a bath maintained at $38^{\circ} \mathrm{C}$., $\mathrm{pH} 7.35$ and aerated with 95 per cent oxygen and 5 per cent carbon dioxide. The composition of the bath in millinnoles is as follows: $\mathrm{NaCl}, 118.9 ; \mathrm{KCl}, 4.7 ; \mathrm{KH}_{2} \mathrm{PO}_{4}, 1.2$; $\mathrm{MgSO}_{4}, 1.2 ; \mathrm{NaHCO}_{3}, 14.9$; dextrose, $5.6 ; \mathrm{CaCl}_{2}$, 2.5 ; sucrose, 49.9 ; and calcium disodium versenate, 0.026 . Contractions of the muscle strips were recorded either isotonically, on smoked paper, or isometrically by means of a strain gauge tension transducer (Grass Model FT03). It has been noted that during a 2 hour incubation period the electrolyte composition and the responsiveness of such preparations reaches a stable level which persists for at least 6 hours.' $^{\prime}$

Similar strips of smooth muscle tissue from resistance vessels (200 to $300 \mu$ diameter) were prepared by (1) threading a fine wire through the lumen of the vessel, (2) mounting the wire in the chucks of a jeweler's lathe, and (3) cutting a helical strip from the vessel wall with a razor blade mounted in a slide rest, as the wire rotates. This process is pictured in Figure 4 , and is more fully described elsewhere. ${ }^{2}$ Such strips, $100 \mu$ wide and $1 \mathrm{~mm}$. long; were mounted in the same manner as aortic strips and tension was recorded by means of a strain gauge. Small vessels obtained from the rabbit mesoappendix have been used for most of these studies; preliminary work has also been done on resistance vessels from the lung and brain. In some experiments two strips, one from a resistance vessel and one from a conduit vessel, shared a common bath, thus making possible a comparison of the responses of the two to the stimuli imposed.

\section{Results}

Role of Potassium in the Contractile Response of Smooth Muscle from Large Conduit Vessels: We have made two studies, previously reported, on the effect of potassium on the responsiveness of smooth muscle from large vessels. In one ${ }^{3}$ it was noted that the response of the isolated rabbit aorta strip to epinephrine stimulation varied directly with the potassium concentration in the bath, over a range of 0 to $9.4 \mathrm{mM}$ 


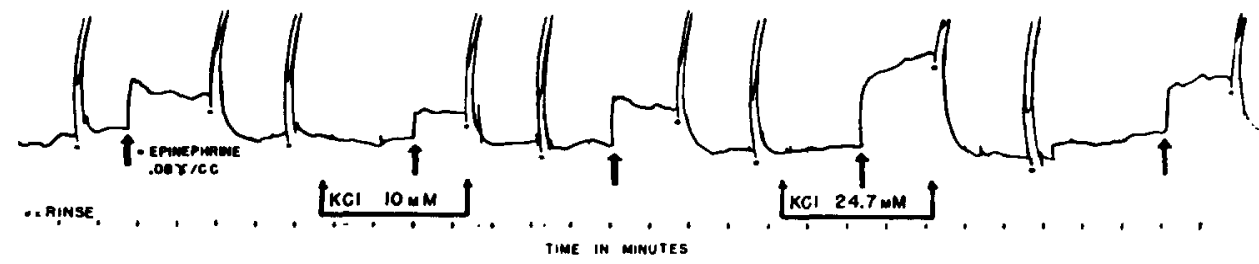

Frg. 5. Effect of high potassium on response of resistance vessel to epinephrine. Note the biphasic effect, a depression at $10 \mathrm{mM}$ and a potentiation at $24.7 \mathrm{mM}$.

concentration. In a study reported just prior to this publication, Leonard ${ }^{4}$ had noted a somewhat greater response of rabbit carotid tissue to electrical stimulation after the removal of potassium from the bath.

Our second study ${ }^{1}$ was a more detailed analysis of the relationship between potassium and vascular responsiveness designed in part to resolve the apparent discrepancy between our results and those of Leonard. The basic objective was to differentiate between the effects of (1) intracellular potassium concentration, (2) extracellular potassium concentration and (3) the ratio between the two. Muscle strips (dog carotid) were stored at $+4^{\circ} \mathrm{C}$. for 48 hours; at the end of this time the intracellular potassium concentration was in equilibrium with that of the surrounding bath. Strips were then mounted in a bath at $38^{\circ} \mathrm{C}$. and responses to electrical stimulation were recorded at 15 minute intervals until a maximum uniform responsiveness was reached. Strips so treated were analyzed for potassium concentration and extracellular water volume, and intracellular potassium concentration was calculated. Such studies were carried out with tissue in baths of different potassium concentrations (from 0 to $24 \mathrm{mM}$ ). It was possible thereby to associate both changes in vascular responsiveness and changes in baseline tension with each of the three parameters: $\left(\mathrm{K}_{\mathrm{i}}\right)$, $\left(\mathrm{K}_{\mathrm{o}}\right),\left(\mathrm{K}_{\mathrm{i}}\right) /\left(\mathrm{K}_{\mathrm{o}}\right)$. The concentration of intracellular potassium was always greater when the amount of potassium in the bath was greater. Just the opposite was true for the ratio of internal to external potassium: when the concentration of potassium in the bath was highest $(24 \mathrm{mM})$ the $\mathrm{K}_{\mathrm{i}} / \mathrm{K}_{\text {o }}$ ratio rose most slowly and reached the lowest steady state value. The following conclusions were reached in these studies: (1) an increase in intracellular potassium increases contractility, (2) an increase in intracellular potassium increases the rate of relaxation, (3) excitability is decreased by a $\mathrm{K}_{\mathrm{i}} / \mathbf{K}_{\text {o }}$ ratio above or below the optimal ratio,
(4) the degree of tonic shortening depends on the $K_{i} / K_{o}$ ratio and (5) contracture develops in a potassium-free environment. The results on which these conclusions are based constitute strong evidence that the potassium ion can influence vascular smooth muscle response both through its gradient across the cell membrane and by the direct action of its intracellular concentration. Furthermore, there is evidence that optimal $K_{i}, K_{o}$ and $K_{i} / K_{o}$ values exist and that when the levels are changed in either direction from the optimal, the performance of the muscle is impaired.

Role of Potassium in the Contractile Response of Smooth Muscle from Resistance Vessels: In earlier experiments with conduit vessels it had been noted that whenever the potassium concentration of the bath was increased above a physiologic level, the magnitude of the response to epinephrine was increased. The pattern of the effect of an increase in potassium concentration of the bath on the response of the resistance vessels to epinephrine stimulation is much more complex. The most common pattern is shown in Figure 5. When the potassium concentration of the bath was increased to $10 \mathrm{mM}$, the contractile response to epinephrine was less than that at control values $(4.7 \mathrm{mM})$. When the potassium concentration was further increased to $24 \mathrm{mM}$, however, the expected potentiating effect of an elevated potassium concentration was evident. Although this is the usual pattern, in some preparations which have been studied the epinephrine response was potentiated at all levels of increased potassium concentration and in other preparations the epinephrine response was depressed at all levels of elevated potassium concentration. These observations constitute further evidence of at least a dual site of action of potassium, depressing at one site and potentiating at the other.

The implied difference between the smooth muscle of large and small blood vessels with regard to the effect of increased potassium con- 


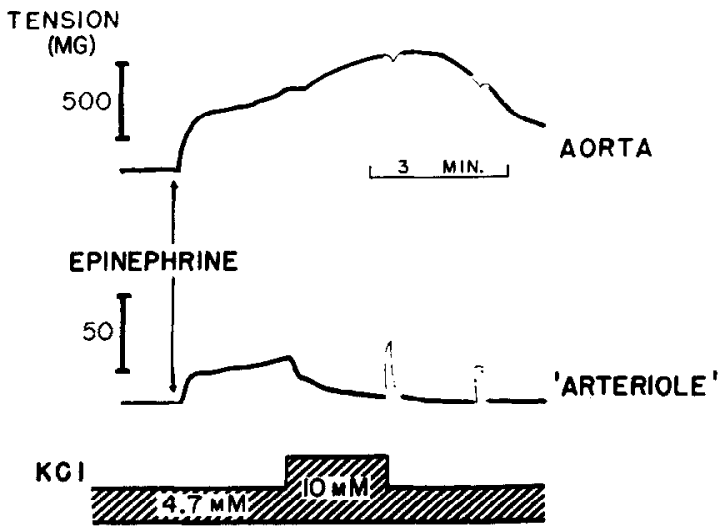

Fra. 6. Comparison of effects of high potassium on response of aorta and "arteriole" to epinephrine. Ten millimoles of potassium caused a potentiation of the aortic response and a depression of that of the resistance vessel.

centration on the epinephrine response is emphasized by the result depicted in Figure 6 . In this experiment, 3 minutes after the initiation of an epinephrine response the potassium concentration of the bath was increased to 10 mM. "Arteriole" and aorta were in the same bath. It is evident that in this preparation the increased potassium concentration caused a relaxation of the epinephrine contraction in the resistance vessel while it potentiated the epinephrine response in the aortic smooth muscle.

The difference in the effect of potassium on the two types of vascular smooth muscle is further emphasized in Figure 7. When potassium was added to the bath to give a final concentration of $25 \mathrm{mM} / \mathrm{L}$ or greater it caused contraction of the smooth muscle from both the resistance vessel and the aorta. However, two striking differences are evident between the responses of these two tissues. The response of the resistance vessel was rapid even at threshold concentrations while that of the aorta was slow and persisted throughout the observation period. The second difference is more obvious with strips in higher concentrations of potassium, where it is evident that the initial rapid contraction of the smooth muscle from the resistance vessels is interrupted by a secondary partial relaxation.

Effect of Steroids on the Response of Vascular Smooth Muscle to Epinephrine: In studies reported elsewhere ${ }^{3,5}$ we have noted that when desoxycorticosterone was present in the bath in low concentration $\left(10^{-7}\right.$ to $\left.10^{-5}\right)$, which caused no shortening of the aortic smooth muscle, it produced a potentiation of an epinephrine

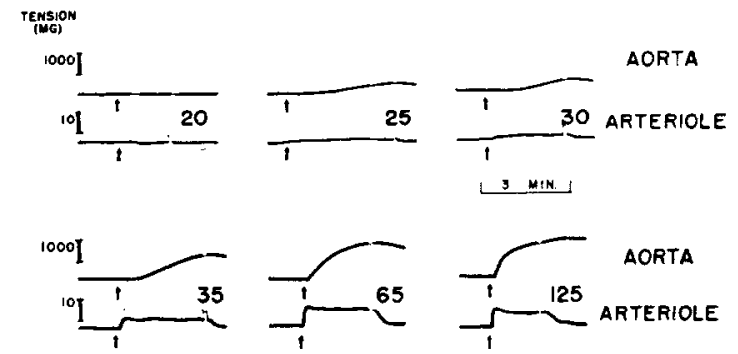

FIc. 7. Comparison of responses of aorta and "arteriole" to $\mathrm{KCl}$; arrows indicate addition of $\mathrm{KCl}$ to the bath; large numbers indicate final $\mathrm{KCl}$ concentration in $\mathrm{mM} / \mathrm{L}$. (See text for significant differences.)

response. Six steroids were compared for their ability to produce this effect. Since different steroids have well defined effects on both electrolytes and organic metabolism, it might be expected that such a comparative study would give some clue to the means by which desoxycorticosterone is capable of producing a potentiation of the response of the aortic smooth muscle. Furthermore, if there were a parallelism between the potentiating ability of a particular steroid and its ability to induce experimental hypertension, such an observation would constitute indirect evidence that the agent could produce the hypertension by sensitizing the vascular smooth muscle to adrenergic responses. However, the results indicated that the relative potentiating action of the various steroids bore no relation to their known physiologic action or to their ability to produce hypertension.

Steroid potentiation of the epinephrine response was also demonstrated by a further contraction of the rabbit aorta when desoxycorticosterone was added during the plateau of an epinephrine response. Doubling the physiologic concentration of potassium produced a similar increase in response to epinephrine. As in the case of desoxycorticosterone, this concentration does not produce contraction of a relaxed aortic strip in the absence of epinephrine. We believe that the response of the aortic strip to epinephrine can be enhanced by decreasing (within limits) the ratio of potassium inside to potassium outside of the vascular smooth muscle cell. When potassium in the bath is increased, this ratio decreases immediately and the potentiation is completed very rapidly. However, when desoxycorticosterone is added the potentiation is very gradual. This is the type of potentiation that would be expected if it were paralleling a 

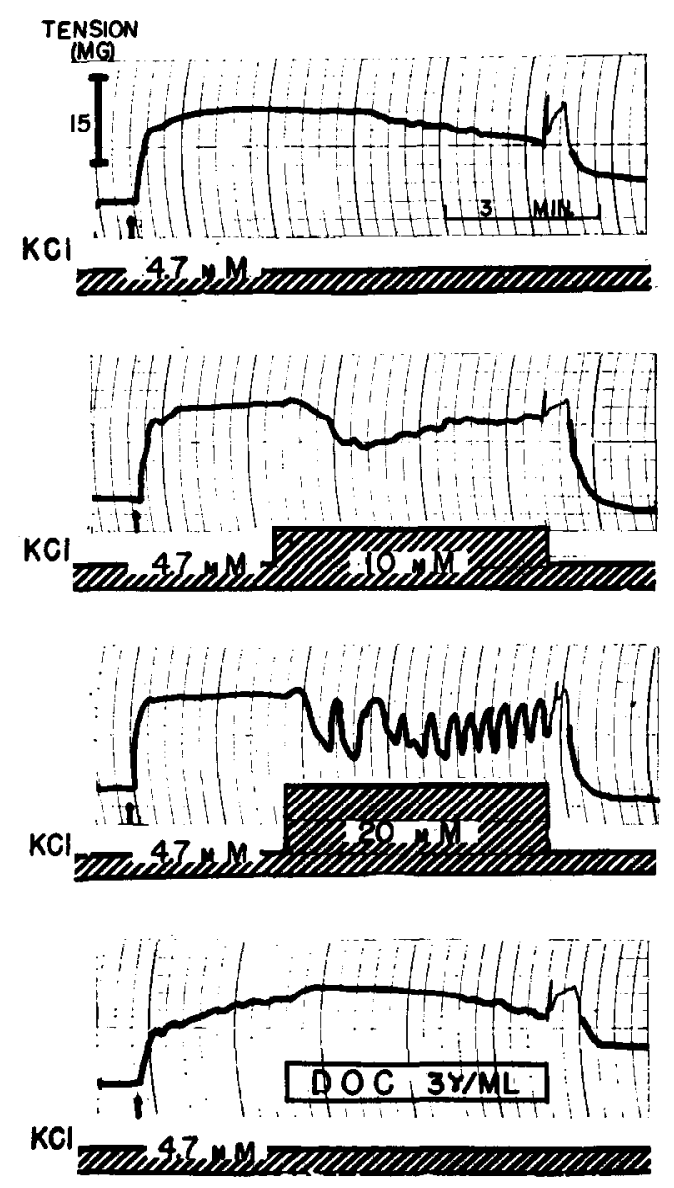

\section{$t=$ EPINEPHRINE $25 \gamma /$ LITER}

FIG." 8. Comparison of the effects of $\mathrm{KCl}$ and DOCl on response of resistance vessel to epinephrine.

gradual and progressive decrease in $\mathbf{K}_{\mathrm{i}} / \mathbf{K}_{\mathbf{o}}$ ratio. Since desoxycorticosterone is known to interfere with the entry of potassium into some cells, ${ }^{6}$ there is some basis for the hypothesis that the mechanism of potentiation by the steroids is a gradual fall in intracellular potassium concentration, thus reducing the $\mathrm{K}_{\mathrm{i}} / \mathrm{K}_{\text {。 }}$ ratio.

In view of our observations that an elevation in potassium concentration frequently reduced the response of the arteriolar smooth muscle, it seemed of interest to explore the action of desoxycorticosterone on the epinephrine response of these resistance vessels. Figure 8 is the record of such a study in which the typical depression of an epinephrine response was observed when the potassium concentration of the bath was increased. No such depression of an epinephrine response was observed when desoxycorticosteronc was added to the same muscle preparation. This use of deoxycorticosterone as a clue for the positioning of a fragment of the jigsaw puzzle, relating the effect of potassium to the contractile process of vascular smooth muscle, demonstrates mainly that the relationships are extremely complex and that there is great individuality between the responses characteristic of vascular smooth muscle from different sites.

Effect of Sodium and Calcium on the Response of Vascular Smooth Muscle to Epinephrine: In an earlier study ${ }^{3}$ we have demonstrated that an increase in sodium concentration in the surrounding bath causes a depression of the vascular smooth muscle response to epinephrine, whereas a decrease in sodium concentration below that of the normal bath results in a potentiation of the response. Recently we have observed similar effects in the response of smooth muscle from the resistance vessels to shifts in sodium concentration in the bath (Fig. 9); the potentiation associated with a decrease in sodium concentration is evident.

The requirement for calcium in the response of vascular smooth muscle to epinephrine seems to parallel its essential role in the contractile process of other muscle tissue. In Figure 10 it is evident that lack of calcium causes progressive decrease in response of smooth muscle from both the aorta and the resistance vessels. Much evidence has been advanced ${ }^{7}$ associating the calcium ion with the coupling between the membrane excitatory process and the contractile protein. It has further been suggested ${ }^{8}$ that in this coupling process, calcium cations compete with sodium cations for anionic binding sites. A decrease in sodium would make more of these anionic sites available to calcium and therefore enhance the contractile response. This hypothesis is in keeping with our observations on the effect of various sodium concentrations on the response of vascular smooth muscle. Admittedly such an interpretation is indirect; however, it attracts us as the only interpretation related to basic observations on the contractile process of muscle tissue which is in keeping with the common observation that the constrictor response of smooth muscle is potentiated by a decrease in sodium concentration. ${ }^{9}$ Interpretations which relate the low sodium potentiation to a reduction in the $\mathrm{Na}_{0} / \mathrm{Na}_{i}$ gradient ${ }^{10}$ have no basis in our current understanding of the contractile process of muscle. 


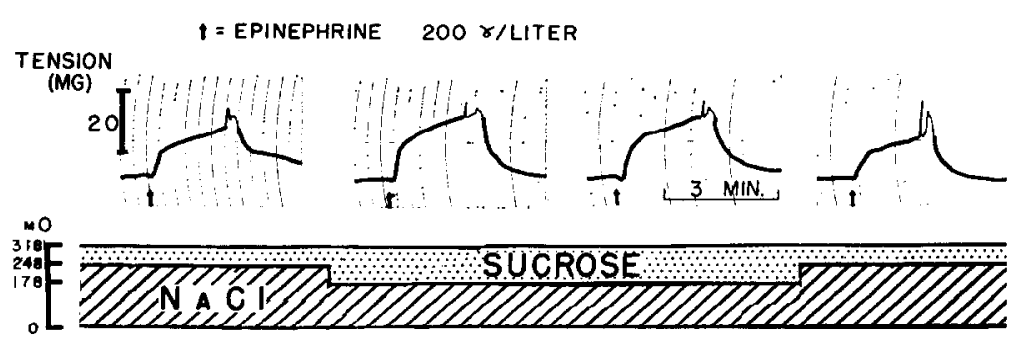

FIG. 9. Effect of low sodium on response of resistance vessel to epinephrine. The response is potentiated by a reduction in sodium concentration.

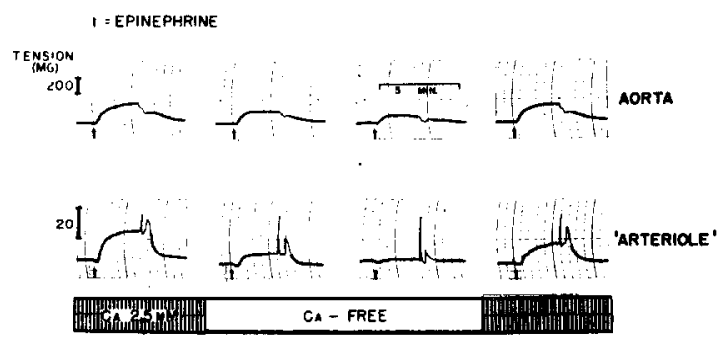

Fig. 10. Effect of no-calcium on response of aorta and "arteriole" to epinephrine. The response of both is depressed in the absence of calcium.

\section{Conclusions}

Finding the answer to any question must begin with the development of an understanding of the problems involved. It will be of great help in positioning the electrolyte fragments of the jigsaw puzzle that will picture the contractile process of vascular smooth muscle, to recognize the various components of the over-all contractile process. Major assistance in this understanding can also be found in the rapidly developing area of physiology of other types of muscle. This philosophic approach to the solution of the problem must be tempered by an expectation of real or apparent differences, not only between vascular smooth muscle and other muscle, but also among vascular smooth muscle preparations from different sources. When a mechanical response is used as a parameter for evaluating the effect of a certain ion, it must be realized that this parameter is the result of a chain of events and that the ion involved may influence several of the links of this chain. Evidence cited in the current study suggests that an increase in potassium concentration may enhance the function of one of these links and depress that of another. The net result, evidenced in the contractile response, will depend on the relative influence of the ion on the two links and also on the inportance of the particular link as a "rate limiting" factor in the response.

\section{Summary}

The following observations of the effect of imposed alteration in electrolyte composition on the contractility of vascular smooth muscle emphasized the complexity of the processes involved:

1. Studies based on the relation of changes in mechanical performance in response to changes in intracellular potassium $\left(\mathrm{K}_{\mathrm{i}}\right)$ and in the $K_{i} / K_{o}$ ratio constitute strong evidence that the potassium ion can influence vascular smooth muscle response both through its gradient across the cell membrane and by direct action of its intracellular concentration.

2. While an increase in potassium concentration in the bath causes only an increase in the magnitude of response of smooth muscle from large conduit vessels, the effect of an increase in potassium concentration on the response of resistance vessels is usually biphasic, small increases depressing and larger increases potentiating the response. These observations constitute further evidence of at least a dual action of potassium.

3. Six steroids were compared as to their ability to produce a potentiation of an epinephrine response. The relative potentiating action bore no relation to their known physiologic effects or to their ability to produce hypertension.

4. A decrease in sodium concentration in the bath causes an increase in the responsiveness of smooth muscle from both conduit and resistance vessels.

5. A decrease in calcium in the bath results in a decrease in the responsiveness of smooth muscle from both of these vessels. 


\section{Aaknowledgment}

We are indebted to Dr. Ruth McVaugh for her editorial contributions which converted our rough ideas to communicable concepts.

\section{REFERENCES}

1. Barr, L., Bohr, D. F. and Headings, V. E. Recovery of carotid artery strips from cold storage. Fed. Proc., 19: 104, 1960.

2. Bohr, D. F., Goulet, P. and TAquini, A. C., JR. Direct tension recording from the smooth muscle of resistance vessels from various organs, Angiology. In press.

3. Bohr, D. F., Brodie, D. C. and Cheu, D. H. Effect of electrolytes on arterial muscle contraction. Circulation, 17: 746, 1958.

4. LEONARD, E. Alterations of contractile response of artery strips by a potassium-free solution, cardiac glycosides and changes in stimulation frequency. Am. J. Physiol., 189: 185, 1957.

5. Bohr, D. F. and Cummings, G. Comparative potentiating action of various steroids on the contraction of vascular smooth muscle. Fed. Proc., $17: 17,1958$.

6. Stolkowski, J. and Reinberg, A. Les modalités de l'appauvrissment des cellules en potassium sous l'action de la désoxycorticosterone. Arch. sc. physiol. 8: 51, 1954.

7. Botrs, J. Triggering of contraction in skeletal muscle. In: Physiological Triggers and Discontinuous Rate Processes, p. 85. Edited by Bullock, T. H. Washington, 1957. American Physiology Society.

8. Niedergerke, R. The "staircase" phenomenon and the action of calcium on the heart. J. Physiol., $134: 569,1956$.

9. Friedman, S. M., Jamieson, J. D. and Friedman, C. L. Sodium gradient, smooth muscle tone, and blood pressure regulation. Circulation Res., $7: 44,1959$.

10. RAAB, $W$. Transmembrane cationic gradients and blood pressure regulation. Interaction of corticosteroids, catecholamines and electrolytes on vascular cells. Am. J. Cardiol., 4: 752, 1959. 